УДК ОО $4+658$

$10.17213 / 2075-2067-2021-3-238-253$

\title{
КАК ПОВЫСИТЬ КОНКУРЕНТОСПОСОБНОСТЬ МНОГОФУНКЦИОНАЛЬНЫХ ТОВАРОВ НА ВНУТРЕННЕМ И ВНЕШНЕМ РЫНКАХ (НА ПРИМЕРЕ ПРОГРАММНЫХ ПРОДУКТОВ)
}

\author{
(C) 2021 г. Г. Н. Хубаев \\ Ростовский государственный экономический университет (РИНХ), \\ 2. Ростов-на-Дону, Россия
}

Целью исследования является разработка методического и инструментального обеспечения для укрепления конкурентных рыночных позиций фирм-разработчиков программных продуктов и повышения конкурентоспособности многофункииональных товаров на внутреннем и внешнем рынках.

Методологическую базу исследования представляют экономическая теория, идеи и рекомендации по совершенствованию рыночной экономики, содержашцеся в работах Мориса Алле (M. Allais) и Фредерика Хайека (F. Hayek), экономико-математические методь.

Результаты исследования. Выявлены противоречия, характерные для российского рынка программных продуктов, сформирована оригинальная по составу совокупность операций для укрепления конкурентных рыночных позиций фирм-разработчиков программных продуктов, предложены методы и инструментальные средства, которые впервые обеспечивают возможность выполнять количественную оценку характеристик потребительского качества программных продуктов, способствуя росту функционального спроса, и позволяют повысить конкурентоспособность многофункциональных товаров-программных продуктов на внутреннем и внешнем рынках.

Перспектива исследования состоит в том, что предложенное в прочессе исследований методическое и инструментальное обеспечение прочессов повышения конкурентоспособности программных продуктов обладает новизной и прикладной полезностью и может использоваться при разработке субъектами рынка многофункииональных товаров в различных предметных областях, способствуя ускоренному развитию экономики страны.

Ключевые слова: программные продукты; многофункциональные товары; сегментация рынка; потребительское качество; активные эксперименты; совокупная стоимость владения.

\section{HOW TO INCREASE THE COMPETITIVENESS OF MULTIFUNCTIONAL PRODUCTS \\ IN THE DOMESTIC AND FOREIGN MARKETS (USING THE EXAMPLE OF SOFTWARE PRODUCTS)}

\author{
(C) 2021 G. N. Khubaev
}

Rostov State University of Economics (RSUE), Rostov-on-Don, Russia

The purpose of the study is to develop methodological and instrumental support for strengthening the competitive market positions of software development firms and 
increasing the competitiveness of multifunctional products in the domestic and foreign markets.

The methodological basis of the research is economic theory, ideas and recommendations for improving the market economy, contained in the works of Maurice Allais (M. Allais) and Frederick Hayek (F. Hayek), economic and mathematical methods.

The results of the study. The contradictions characteristic of the Russian software market are revealed, an original set of operations is formed to strengthen the competitive market positions of software development firms, methods and tools are proposed that for the first time provide an opportunity to perform a quantitative assessment of the characteristics of the consumer quality of software products, contributing to the growth of functional demand, and allow to increase the competitiveness of multifunctional products-software products in the domestic and foreign markets.

The perspective of the research is that the methodological and instrumental support for the processes of increasing the competitiveness of software products proposed in the research process has novelty and applied utility and can be used in the development of multifunctional goods by market participants in various subject areas, contributing to the accelerated development of the country's economy.

Key words: software products; multifunctional products; market segmentation; consumer quality; active experiments; total cost of ownership.

Введение. Напомним, что «конкурентоспособность товара - способность выдерживать конкуренциию... Параметр конкурентоспособности товара - качество. Это совокупность свойств товара, которые являются условиями его пригодности в удовлетворении определенных потребностей согласно его назначению» [1]. Однако, как отмечено в [2], «российские специалисты в области информационных технологий высоко иенятся во всем мире. Но лишь единииьы из тысяч ИТ-продуктов, разработанных в нашей стране, успешно продаются на глобальном рынке». Неудивительно, что и ничтожно мало поступлений в доходную часть бюджета страны от экспорта ИТ-продуктов. Одновременно в России «продолжает снижаться доля разработки программного обеспечения - в 2019 г. она составила $10 \%$ против 14\% в 2018 г.» [3], и это при том, что и школьники, и студенты постоянно и очень успешно выступают на международных конкурсах по информатике. Правда, в [4] утверждается, что именно ошибки в выборе приоритетных направлений развития экономики страны привели к ослаблению конкурентных рыночных позиций российского ИТ-бизнеса, и даже сегодняшние реальные усилия государства по финансовому подкреплению индустрии ИТ не оказали заметного влияния на успехи российского ИТ-бизнеса на внешних и внутреннем рынках.

Но почему так происходит, почему не растет столь необходимый для ускоренного сочиально-экономического развития страны объем продаж российских ИТ-товаров? Ведь, как показали наши исследования [5], показатель «ВВП на одного работающего (в долларах)» статистически значимо зависит именно от приращзения объема экспорта услуг ИКТ (за три года, в \% от экспорта услуг - лаг составляет три года; статистические характеристики: $b 1 / \sigma_{\mathrm{b} 1}=23,2 ; R_{\text {ск }}^{2}=0,87$; $F$ кр $=539,9$. Значимость $F$ кр $=1,25 \mathrm{E}-08$; Германия). А показатель «ВВП на душу населения, ППС» зависит от приращчения величины сборов за использование интеллектуальной собственности (за один год, в текущих ценах - лаг составляет один год; статистические характеристики: $b 1 / \sigma_{\mathrm{b} 1}=20,9 ; R^{2}=0,87$; $F$ кр $=436,5$. Значимость $F$ кр $=2,89 \mathrm{E}-08$; Швеция). И, как известно, компьютерная программа, в отличие от большинства других товаров, является практически полностью интеллектуальным продуктом.

Причина такого положения на рынке программных продуктов (ПП), по нашему мнению, достаточно очевидна, ведь спрос на ПП 
и другие многофункциональные товары является функциональныл, т.е. «обусловлен присушими этому благу потребительскими качествами», поэтому покупатель должен иметь возможность сравнивать характеристики потребительского качества представленных на рынке товаров - ПП.

Однако сегодня ситуация на рынке ПП не позволяет потенциальному покупателю сделать обоснованный выбор нужного именно ему ПП, т.е. покупатель, придя на внутренний или внешний рынок за покупкой ПП, не сможет сравнить представленные на рынке ПП по: *функциональной полноте; *затратам времени и других ресурсов на выполнение нужных ему функций, *затратам времени и труда на изучение-освоение Руководства пользователя (коэффициент вариации таких затрат может быть очень большим); *сравнительному качеству интерфейса ПП; *совокупной стоимости владения ПП и по конкретным численныл значениям других характеристик потребительского качества ПП [6].

Но о какой же рыночной экономике при такой ситуации на рынке ПП может идти речь? Ведь «прозрачность» рынка, сведения о ценах и о потребительском качестве товаров и услуг, информачионное обеспечение покупателей и продавцов является «координируюшим механизмом рыночной экономики», стимулирующим ее саморегуляцию.

В статье, базируясь на ранее выполненных исследованиях, выполнен анализ ситуации на внутреннем рынке ПП, сформирован состав операций процесса укрепления конкурентных рыночных позиций фирмы-разработчика ПП, предложены оригинальные авторские методы и инструментальные средства повышения конкурентоспособности не только поставляемых на внутренний и/или внешний рынки, «коробочных» ПП, но и пригодные для повышения конкурентоспособности многофункциональных товаров различного назначения.

1. О ситуации на внутреннем рынке программных продуктов. Современная цивилизация уже вступила в период формирования информационного общества. Однако в ходе развития и применения информационных технологий неизбежно возникают и на- капливаются серьезные проблемы. Рассмотрим, например, ситуацию, сложившуюся на рынке специализированных ПП, в первую очередь ПП для экономических приложений.

Чем же характеризуется эта ситуация? Как свидетельствует анализ публикаций, во многих случаях покупателю-пользователю предлагаются десятки и сотни программных продуктов одного назначения, отличающихся по качеству и цене. Примечательно, что и цена, и число продаж зачастую мало зависят от реального качества представленного на рынок программного продукта. И при этом лишь ничтожно малое количество ПП можно считать относительно завершенными, рыночными продуктами. С аналогичной ситуацией на рынке ПП покупателям пришлось столкнуться уже достаточно давно: «Программные продукты с идентичныл функциональным наполнением значительно отличаются иеной, причем иногда данное отличие составляет порядок» [7].

Но почему же на рынке ПП имеет место такое негативное развитие событий? Почему продолжается пополнение рынка программными продуктами, не имеющими практически никакой потребительской ценности? Почему покупатели-пользователи продолжают расходовать время и деньги на приобретение, освоение и внедрение заведомо неэффективных ПП?

Попытаемся объяснить причины возникновения аномальной ситуации на рынке ПП. Представляется достаточно очевидным, что если бы разработчик ПП заранее, до начала проектирования, имел возможность получить достоверные данные о потребительских свойствах существующих программных продуктов или же сам мог оперативно количественно оценить качество существующих ПП, то вряд ли на рынке появились бы сотни и тысячи несопоставимых по качеству компьютерных программ одного назначения.

Аналогичная ситуация и с покуnаmeлем ПП. Если бы покупатель либо независимая фирма-посредник могли количественно оценивать потребительское качество ПП, проводить экспресс-оценку достоверности информации, содержащейся в рекламных проспектах на ПП, то, несомненно, было бы значительно меньше ошибок при покупке программ для конкретных приложений. Ведь 
сравнение ПП, включая новые разработки, проводится, как правило, на качественном уровне, без использования количественных экономических критериев и без соотнесения эксплуатационных характеристик ПП с особенностями конкретной предметной области, с набором потребностей конкретного пользователя. Как отмечено в [7]: «Наполнение рынка... новыми ПП... становится серьезным препятствием для пользователей, поскольку в широком спектре программных продуктов без соответствующей информационной поддержки достаточно трудно ориентироваться..., а увеличение количества предлагаемых разработок разных классов и функциональной направленности возводит перед пользователями информационный барьер, преодолеть который самостоятельно не представляется возможным».

Получается, что сегодня ни разработчики ПП, ни пользователи, ни фирмы-посредники не могут корректно, обоснованно сравнить качество существующих ПП по экономическим критериям, оценить, насколько один ПП эффективнее другого.

Покажем, что такая ситуация на рынке ПП является аномальной, она не выгодна ни продавцу, ни покупателю, разорительна для общества в целом. Начнем с продавца.

Bo-nepвыx, в отличие от продукции материального производства тиражирование компьютерных программ требует ничтожных затрат времени и средств по сравнению с затратами на разработку, поэтому спрос на тот или иной ПП даже при наличии на рынке всего лишь одного нужного покупателям программного продукта может быть быстро удовлетворен. Совершенно очевидно, что в таких условиях каждый вновь разрабатываемый ПП для обеспечения ему устойчивой рыночной позиции должен либо лучше, чем существующие, удовлетворять потребности потенциальных пользователей, либо быть эффективнее существующих (дешевле, проще в эксплуатации, легче в освоении и т.д.). Естественно, что производить и предлагать рынку ПП с заведомо худшими характеристиками потребительского качества никакой разумный продавец-разработчик, казалось бы, не должен: слишком велики затраты, слишком велик риск, что эти затраты окажутся напрасными.
Bo-вmopыx, общество в целом также несет прямые и косвенные убытки, ведь созданный, но не нужный покупателю ПП не имеет остаточной потребительской стоимости. При этом общество не получает отдачи от интеллектуальных и финансовых ресурсов, отвлеченных из других сфер приложения и использованных на разработку ПП.

Теперь обратимся к другому участнику процесса товарного обмена - к фирме-покуnameлю ПП. Для фирмы-покупателя неудачный выбор ПП обернется убытками, которые обусловлены:

1) затратами денежных средств и времени на приобретение оказавшегося неэффективным, ненужным программного продукта, на его освоение и внедрение, включая создание БД, справочников и т.д.;

2) потерями ожидаемого эфрфекта от купленного ПС (не получена отдача на вложенный капитал);

3) дополнительными текущзими расходами на эксплуатаџию ПП (если она была начата);

4) потерями темпа в конкурентной борьбе при отказе от уже купленного ПП и повторными расходами времени и средств на приобретение, освоение и внедрение нового ПП того же назначения. Правда, здесь опять нет никакой гарантии, что вновь приобретенный ПП окажется лучше прежнего (о моральных потерях, которые несет покупатель, мы здесь уже не говорим).

Легко представить, насколько велики прямые убытки от ошибочных решений на стадии разработки, покупки и внедрения программных продуктов, учитывая, что *трудоемкость разработки ПП средней сложности может достигать десятков и сотен человеко-лет, а рыночная цена колебаться от десятков до десятков тысяч долларов; *затраты времени на освоение и внедрение многих специализированных ПП составляют 1-3 года; *затраты на сопровождение специализированных ПП в ряде случае соизмеримы с затратами на разработку.

И при этом теоретический объем рынка для некоторых ПП измеряется сотнями тысяч единиц. Тогда экономия только на эксплуатационных расходах и только на ПП одного назначения может обернуться в масштабах страны дополнительными десятками милли- 
онов долларов в год (!). А реализовав для ПП одного назначения нечто подобное [8], можно существенно сократить огромные потери $и н$ теллектуальных и финансовых ресурсов.

[Замечание. Если для стимулирования ускоренного развития отрасли ИТ государство будет снижать налоги предприятиям (юрлицам), ориентированным на разработку рыночных ПП, то такое решение еще больше затруднит потенциальному покупателюпользователю поиск ПП, отвечающего его требованиям. Ведь предприятия, чтобы снизить величину налогообложения, будут массово разрабатывать ПП одного назначения с заведомо худшими характеристиками потребительского качества. Благоприятный для страны выход из подобного затруднительного положения может быть, по-видимому, один: создание нескольких конкурирующих фирм, функционирующих под контролем государства, выполняющих сравнительную количественную оценку потребительского качества предлагаемых рынку ПП и предоставляющих результаты оценки в открытом доступе (см., например, [9]).]

\section{2. Повышение конкурентоспособности} программных продуктов на внутреннем и внешнем рынках: методическое и инструментальное обеспечение. Как отмечено нами ранее, фирмы-продавцы ПП, чтобы заинтересовать потенциальных покупателей и с большей вероятностью продать свой товар, должны обеспечивать покупателей достоверной информацией о потребительском качестве и конкретных преимуществах разработанного ими ПП. Очевидно, что поэтому продавец долюсен правильно:

1) Выбрать «прибыльный» сегмент рынка и оценить величину спроса на многофункциональный товар-ПП, который будет представлен в выбранном сегменте рынка. Для оптимального выбора рыночной ниши необходимо определить, в чем нуждается потребитель, как осуществить сегментирование рынка и какой сегмент рынка будет обслуживать фирма «при проникновении на рынки, характеризуемые высокой степенью конкуренции». Однако сделать это непросто, ведь с развитием научно-технического прогресса усложняются и предлагаемые рынку товары и услуги - автомобили, само- леты, смартфоны, компьютеры, программные продукты. Так, число реализуемых функций у многих рыночных продуктов исчисляется сотнями и тысячами. Растет и число модификаций товаров одного назначения.

Спрашивается, как вступающей на рынок фирме выбрать свою нишу, свой сегмент на целевом рынке, осуществить многосегментную дифференциацию рынка? И как потенциальному покупателю найти среди множества сопоставимых конкурируюших продуктов нужный именно ему товар с нужными именно ему функциями, товар нужного качества, простого в освоении, с минимальными затратами ресурсов на обслуживание и сопровождение, доступного по цене и т.д.? Причем желательно выбрать оперативно, с минимальными затратами времени и других ресурсов.

Кроме того, фирме, проектирующей коробочный ПП, также важно знать, какие из интересующих потенциального покупателя функиий еще не может реализовать ни один из рыночных ПП (ведь тогда цена разрабатываемого этой фирмой продукта могла бы стать квази-монопольной).

Однако сегодня, насколько нам известно, ни одна из существующих методик не может дать ответ на перечисленные вопросы.

В [10] предложена авторская методика, позволяющая корректно сравнивать представленные на рынке программные продукты по функциональной полноте, осуществлять многосегментную дифференцииацию рынка, выявлять, какие конкретные функции ПП интересуют разныле группь покупателей.

Методика базируется на использовании программно-реализованных оригинальных алгоритмов для *оценки функцииональной полноты товаров и *пошагового уточнения ранжирования функций потенциальными покупателями товара. Апробация и программная реализация используемых алгоритмов подтвердили их прикладную полезность и возможность использования в любой отрасли производства, в любой предметной области.

Отличие метода «Оценка функциональной полноты объекта (ФП-метод)» $[11,12]$. Метод ориентирован на оценку информационного веса групп признаков, а для оценки сходства и различия между объектами по составу признаков используются операции 
с множествами: пересечение, разность, объединение, отношение и мера подобия Жаккарда, причем для наглядного представления взаимосвязей между объектами по выбранным признакам используются графы, построенные по логическим матрицам включения (поглощения).

Преимущества ФП-метода. Метод позволяет с минимальныли затратами времени, финансовых и интеллектуальных ресурсов: * систематизировать сведения о составе признаков у рассматриваемых объектов (существующих и/или проектируемых); *количественно оценивать степень соответствия состава признаков конкретного объекта требованиям пользователя; *реализовать упорядочение объектов по критерию полноть состава признаков; *на стадии предварительного анализа исключить из дальнейшего рассмотрения объекты, у которых отсутствуют нужные пользователю признаки; *расширить для потребителя-пользователя возможности оптимального выбора на рынке нужных ему объектов-товаров, предоставив перечень признаков (потребительских свойств) каждого товара, а разработчикам дать возможность определять направления совершенствования созданного ими объекта; *сформировать группу объектов, имеющих одинаковый состав признаков, сопоставить их цены и другие характеристики потребительского качества.

При использовании метода (его программной реализации) появляется возможность с минимальными затратами ресурсов получать сравнительную количественную оценку функциональной полноты ПП любой сложности, содержащих, например, свыuе 300 (Е. Пятина), 500 (Е. Пахомов), 900 (С. Широбокова) функций. Рассмотренные процедуры сравнения применимы в любой отрасли производства, в любой предметной области.

На следующем этапе сегментирования рынка необходимо выявить и упорядочить функции товара, которые нужны конкретному подмножеству потенциальных покупателей. Для этого целесообразно использовать метод пошагового уточнения ранжирования объектов (ПУРО), описанный в [13]. Метод основан на интегращии дельфийской процедуры прогнозирования будущего (разработан
О. Хелмером, Н. Долки, Т. Дж. Гордоном) с предложенной Дж. Кемени (J.G. Kemeny) оценкой медианы и расстояния между упорядочениями объектов. Отличие метода ПУРО: *дельфийская процедура используется при ранжировании экспертами характеристик-функиий объектов (а не для прогнозирования будущего); *для количественного анализа степени сходимости мнений экспертов после каждого тура опросов, выявления согласованных групп экспертов и оценки иелесообразности завершения экспертизы используется расстояние Кемени (мера близости на отношениях линейного порядка), а в качестве результирующего ранжирования - медиана Кемени; *для группировки участников экспертного опроса, у которых могут оказаться схожими взгляды относительно вариантов ранжирования рассматриваемых характеристик, устанавливаются пороговые значения расстояния Кемени между ответами экспертов, исходя из вероятности совпадения их мнений, например, близкой к $0.8 ; 0.9 ; 0.95 ; 0.99 ;$ *для поиска в каждой из образовавшихся групп экспертов согласованного с членами группы ранжирования (упорядочения) характеристик рассчитывается медиана Кемени.

Преимущества метода ПУРО: *корректность метода (теорема о корректности доказана в 1978 году — «медиана Кемени единственное результирующее строгое ранжирование, являющееся нейтральным, согласованным и кондорсетовым»); *повышение точности результатов экспертизы за счет наличия обратной связи при реализации каждого последующего тура; *при использовании пошагового уточнения ранжирования характеристик сохраняются известные преимущества и дельфийской процедуры (анонимность и возможность ознакомления с объяснениями, представленными в защиту сильно отличающихся ответов), и алгоритма поиска согласованного упорядочения объектов - корректный расчет медианы и расстояния Кемени; * совместное использование и дельфийской процедурьи, и предложенного Кемени корректного подхода к упорядочению функций позволило: получить содержательно приемлемый количественный критерий для обоснованного завершения экспертизы - устанавливается определенная 
величина относительного (например, 5\%) изменения суммарного расстояния Кемени; формировать группы экспертов, ориентируясь на выбранные (в зависимости от степени согласованности мнений) пороговые значения расстояния Кемени и исследовать причины взаимосвязи оценок экспертов; находить согласованное с членами каждой группы экспертов упорядочение характеристик, рассчитав точно или приближенно медиану Кемени; *метод пошагового уточнения ранжирования характеристик объектов, будучи простым и корректным, не имеет ограничений на количество участников экспертного опроса.

2) Определить спрос на проектируемый ПП и цену, которую согласятся заплатить покупатели ПП. Прогнозирование спроса и цены ПП можно осуществить, воспользовавшись методом [14-16], который ориентирован на пошаговое уточнение значений показателя с оценкой характеристик распределения (ПУЗ-ОХР).

Отличие метода ПУЗ-ОХР: *интеграция дельфийской процедуры, обычно используемой для прогнозирования будущего, с экспертизой, направленной на получение значения искомого показателя; * специалистам, участвующим в экспертизе, предоставляется возможность рассматривать возражения и предложения других членов экспертной группы в атмосфере, свободной от влияния личных качеств участников, что способствует активизации интеллектуальной деятельности экспертов; *предусмотрено количественное определение момента (номера шага) завершения экспертного опроса (по величине изменения коэффициента вариации); * реализация обратной связи в процессе экспертизы путем информирования специалистов-экспертов о результатах предыдущего шага; * использование программы последовательных шагов, на каждом из которых реализуется полный цикл экспертизы; *аппроксимация оценок каждого эксперта, представленных тремя значениями искомого показателя (минимальное, максимальное и наиболее вероятное значение) треугольным распределением, а представленных двумя значениями (минимальное и максимальное) - равномерным распределением; *использование имитационного моделирования для определения обобщенного коллективного мнения $\boldsymbol{n}$ экспертов как среднего $\boldsymbol{n}$ случайных величин, имеющих треугольное или равномерное распределения (мнений $\boldsymbol{n}$ участников экспертной группы); * получение оценок статистических характеристик (математического ожидания, дисперсии, коэффициента вариацчии, медианы, эксиесса, асимметрии) и распределения значений искомого показателя (в виде таблицы и гистограммы); *возможность определения вероятности того, что значения показателя не превысят конкретную величину или попадут в заданный диапазон значений.

Преимущества метода ПУЗ-ОХР: *Повышение точности результатов экспертизы за счет наличия обратной связи при реализации каждого последующего тура, обеспечения эксперту возможности указывать три или два значения искомого показателя, определения по результатам имитационного моделирования вероятности попадания величины показателя в заданный диапазон значений; *уменьшение психологической нагрузки на эксперта и негативного влияния на результаты экспертизы присутствия начальников и/или амбициозных личностей, поскольку сохраняется анонимность, и экспертьл не обшаются друг с другом и не знают, кто дал конкретное обоснование в зашуиту сильно отличаюшихся значений показателя; *представление суммарного распределения как математического ожидания суммы равномерных или треугольных распределений оценок отдельных экспертов в виде гистограмм и таблиц, статистических характеристик распределений позволяет получить результирующее распределение значений показателя даже при большой дисперсии оценок и условии, что эксперты указывают два или три значения; *pacчеm статистических характеристик распределений (математическое ожидание, дисперсия, коэффициент вариации, медиана, асимметрия, эксцесс) и таблиц распределений обеспечивает возможность оценки вероятности попадания значений искомого показателя в заданный диапазон; *⿻blявление самопроизвольных группировок экспертов, оценки которых относительно искомых значений показателя близки, дает возможность исследовать причинь образования таких групп. Формирование групп эк- 
спертов осуществляется, исходя из заданной пороговой величины вероятности конкретного диапазона значений прогнозируемого показателя.

Метод корректен, многократно апробирован, подтвердил свою прикладную полезность в процессе использования в различных предметных областях.

3) Выполнить оценку функциональной полноты (ФП) существующих, представленных на рынке товаров-ПП аналогичного назначения - потенциальных конкурентов создаваемого ПП. Для выполнения сравнительной количественной оценки состава сотен $и$ тысяч характеристик, которыми обладают сотни и тысячи объектов-ПП, потребуются существенные затраты ресурсов. Причем, желательно получить результат оперативно, с минимальными затратами времени и других ресурсов.

В $[11,12]$ предложен метод сравнительной оценки состава функций любых ПП одного назначения. Рассмотренные процедуры сравнения применимы в любой отрасли производства, в любой предметной области.

4) Выявить востребованные потенциальными покупателями-пользователями, но отсутствующие у существующих, представленных на рынке ПП функции (чтобы реализовать эти функции у проектируемого ПП и обеспечить возможность устанавливать квазимонопольную изену при его продаже).

Предположим, что оперативно определен состав функций у всех рыночных ПП-конкурентов. Но как определить, какими новыми функциями целесообразно дополнить состав функций проектируемого «коробочного» ПП? Какую пользу такое расширение состава функций ПП принесет фирме-разработчику программного продукта?

При формировании состава функций проектируемого ПП целесообразно использовать метод пошагового уточнения ранжирования объектов (ПУРО), описанный в [13].

5) Выполнить сравнительный анализ и обоснованный выбор варианта интерфейса ПП из нескольких сопоставимых (варианта внешнего вида товара). Для абсолютного большинства покупателей исключительно актуальной является задача сравнительной оценки и выбора варианта дизайна объекта - варианта интерфейса програм- много продукта, варианта внешнего вида кузова автомобиля и т.п. В [17] предложены корректные процедуры, ориентированные на пошаговое использование расстояния $u$ медианы Кемени и непараметрических методов статистики при сравнении вариантов интерфейса программного продукта, внешнего вида любого художественно оформленного объекта (см. также [18]).

6) Оценить затраты ресурсов на сопровождение проектируемого ПП. Для этого на первом этапе * выделяют из состава представленных на рынке ПП групnу аналогичного с разрабатываемым ПП назначения; *определяют на основе ретроспективных данных затраты ресурсов (трудовых, финансовых) на сопровождение ПП этой группы и сообщают эти сведения участникам экспертного опроса; *выполняют прогнозирование затрат на сопровождение разрабатываемого ПП с использованием пошагового уточнения значений показателя с оценкой характеристик распределения - ПУЗ-ОХР (см. [14-16]).

7) Выполнить сравнительную оценку потребительского качества руководства пользователя (инструкции, справочной системы) по критерию минимума затрат времени покупателя-пользователя на изучение функциональных возможностей ПП. Как известно, затраты времени на освоение нового учебного материала - учебной дисциплины, руководства пользователя, методических рекомендаций и др. зависят от уровня начальной (исходной) подготовки обучаемого, от его способностей, от степени мотивации, от условий обучения, сложности изучаемого материала и других факторов. Задача определения затрат времени на получение знаний, на реальное освоение нового учебного материала исключительно актуальна для всех отраслей общественного производства и науки - ведь в условиях непрерывного ускорения НТП каждый человек вынужден учиться всю сознательную жизнь.

В процессе анализа содержания Руководства пользователя необходимо ответить на ряд вопросов: насколько легко непрофессионалу в области ИТ-технологий понять содержание инструкции для покупателя-пользователя? Как в каждом конкретном случае оценить величину затрат времени на изуче- 
ние Руководства пользователя? Как определить вероятность практического освоения конкретного материала за заданное время? Каковы, наконец, статистические характеристики затрат времени на получение знаний, характеристики распределения времени освоения любого нового учебного материала?

В [19] предложена универсальная методика оценки затрат времени на получение знаний, включающая методы и инструментальные средства для расчета статистических характеристик распределения времени освоения любого учебного материала при различных условиях формирования исходной информации: в проиессе анкетирования обучаемых, по даннылм натурных экспериментов и по результатам экспертных опросов. Статистические характеристики затрат времени (математическое ожидание, дисперсию, коэффициент вариации, эксиесс, асимметрию) и распределение (в виде таблиц и гистограмм) оценивают путем имитационного моделирования. На основании результатов имитационного моделирования легко определить доверительные границьь для конкретного значения затрат времени на изучение Руководства пользователя.

8) Определить затраты времени и трудовых ресурсов на получение интересующего покупателя-пользователя результата, например, на выполнение и каждой функции, и полного состава функций при использовании как представленных на рынке ПП, так и создаваемого ПП (в условиях применения конкретного состава системного программного обеспечения и технических средств).

В [20, 21] предложена оригинальная методика, позволяющая корректно количественно оценивать и сопоставлять затраты на применение ПП одного назначения, сравнивать ПП по экономическим критериям. При использовании методики впервые появляется возможность корректно, более обоснованно и с большей достоверностью осуществлять следующее: *соотносить потребности конкретного пользователя с возможностями существующих и проектируемого ПП; *выделять из числа выполняемых вручную операций те, которые нужно реализовать в новой версии ПП в первую очередь, чтобы минимизировать затраты времени и трудовых ресурсов на решение задачи; *определять, вы- полнение каких машинных операций следует ускорить, например, перепрограммировав, модифицировав структуру БД и т.п.; * рассчитывать экономическую целесообразность автоматизированного выполнения конкретной операции, решения задачи (комплекса задач), сопоставив затраты на ручной и машинный варианты; *выбирать оптимальную структуру БД (информационной системы) в процессе проектирования или модификации, базируясь, например, на результатах формализованного анализа информационных потребностей пользователей и др.

Кроме того, в технической документации абсолютного большинства рыночных ПП отсутствуют сведения, позволяющие оценить выходные характеристики программной системы и их динамику при изменении объемов входной информации, структуры БД, состава комплекса технических средств, СУБД, языка программирования и т.д. Более того, в литературе по проектированию и анализу ИС, ПП и БД практически не описаны способы корректного получения таких данных, определения их прогнозной ценности, доверительных границ для рассчитанных статистических характеристик.

В [22] применительно к проблемам проектирования и анализа ИС, ПП и БД впервые:

*вылвлены и систематизировань важнейшие особенности программных продуктов как объектов активного экспериментирования;

*описаны свойства факторов, влияющих на выходные характеристики ПП. Показано, что большинство факторов являются управляемыли, количественнылми, независимылми; они оказывают непосредственное (прямое) воздействие на исследуемый объект;

*определены основные требования к свойствам моделей, формируемым по результатам активных экспериментов;

* выделены критерии выбора плана эксперимента при исследовании ПП;

* даны рекомендации по применению конкретных планов для постановки экстремальных по выбранныл критериям экспериментов.

9) Оценить степень защищенности проектируемого ПП, рассчитав предварительно ущцерб от кражи интеллектуальной собственности путем копирования текста программы для ЭВМ и от реализации других угроз защиицаемому объекту. Ведь ПП, 
будучи созданным практически полностью в результате интеллектуального труда разработчиков (в отличие от больиинства других товаров) требует определенных, зачастую, значительных затрат ресурсов на защитуу от кражи результата интеллектуального труда, заключенного в ПП.

Важнейшим потребительским свойством программных продуктов для зашиты информации (исполняемых программ, БД, текстовых файлов) является качество выполнения защитных функций. Это качество можно характеризовать величиной среднего значения, дисперсии и законом распределения времени вскрытия защиты, значением вероятности вскрытия защиты за определенное время либо, наоборот, вероятности того, что за заданное время зашита не будет вскрыта.

По аналогии с другими ПП при сравнительной оценке характеристик потребительского качества программных систем защиты информации вся работа по вскрытию (взлому) защиты также может быть представлена в виде последовательности элементарных операций. Для каждой такой операции определяются статистические характеристики и закон распределения времени ее выполнения, а затем с использованием имитационного моделирования или аналитическими методами оцениваются вероятностные характеристики и законы распределения времени решения всей задачи по вскрытию защиты.

Причем основные компоненты методики оценки производительности ПП, т.е. оценки затрат времени пользователя на реализацию функций ПП, остаются неизменными (см., например, [5, 20-25]).

[Замечание. При оценке времени вскрытия защиты ПП от копирования с ориентацией на метод [25] следует учитывать результаты исследований А.М. Костина [26]. Эксперименты со студентами выпускных курсов специальностей ИТ и защиты информации показали, что величина правосторонней асимметрии распределения затрат времени на вскрытие защиты одного и того же ПП разными исполнителями может быть огромной.]

10) Рассчитать величину совокупной стоимости владения (ССВ) программным продуктом. Большинство программных систем (программных продуктов, информационных систем) относят к объектам длительного пользования. В понятие IT-затрат входят затраты, связанные с приобретением, внедрением и использованием ПП. Эти затраты формируют совокупную стоимость владения программной системой (Total Cost of Ownership ТСО), включающей первоначальные и последующие затраты, определяя их как единые затраты на программную систему в процессе ее создания и эксплуатации.

Для расчета ССВ (см. [27, 28]): *выделяют с использованием метода пошагового уточнения ранжирования объектов (ПУРО) упорядоченный перечень основных затрат ресурсов; *определяют, используя метод пошагового уточнения значений показателя с оценкой характеристик распределения (ПУЗ-ОХР), статистические характеристики (математическое ожидание, стандартное отклонение, коэффициент вариации, медиану, асимметрию, эксцесс) и распределение (в виде гистограмм и таблиц) совокупной стоимости владения ПП. Результаты расчета позволяют оценивать вероятность попадания $\mathrm{CCB}$ в конкретный диапазон значений (примеры реальных расчетов ССВ представлены в [28]).

Описанные методы, будучи оригинальнылми и обладая очевидной прикладной полезностью, реализованы в виде программ для ЭВМ и использованы в т.ч. при разработке прикладных программ в различных предметных областях (см., например, [29-35] и др.).

Заключение. В статье впервые:

1. Выявлены и систематизированы противоречия, характерные для российского рынка программных продуктов, сформирована оригинальная по составу совокупность операциий для укрепления конкурентных рыночных позиций фирм-разработчиков программных продуктов.

2. Обосновано применение оригинальных методов и инструментальных средств, которые впервые обеспечивают возможность выполнять количественную оценку характеристик потребительского качества программных продуктов, способствуя росту функционального спроса, и позволяют повысить конкурентоспособность многофункциональных товаров - программных продуктов на внутреннем и внешнем рынках.

3. Созданное автором методическое и инструментальное обеспечение процессов повы- 
шения конкурентоспособности программных продуктов обладает новизной и прикладной полезностью и может использоваться при разработке субъектами рынка многофункциональных товаров в различных предметных областях, способствуя ускоренному развитию экономики страны.

\section{Литература}

1. Большая экономическая энциклопедия. - М.: Эксмо, 2007. - 816 с.

2. Как увеличить спрос на российские ИТ-решения на глобальном рынке? [Электронный ресурс]. - Режим доступа: https:// www.kommersant.ru/doc/3991175.

3. Рынок ИТ: итоги 2019 [Электронный pecypc]. - Режим доступа: https://www. cnews.ru/reviews/rynok_it_itogi_2019.

4. Хубаев Г.Н. Как уменьшить вероятность ошибок при выборе приоритетных направлений социального и экономического развития страны // Бюллетень науки и практики. - 2019. - Т. 5. — №12. - С. 265-280.

5. Хубаев Г.Н. Прогнозирование динамики индикаторов уровня развития экономики страны: модели, методы, инструментальные средства (на примере Германии, России и Швеции) // Вестник ЮРГТУ (НПИ). 2020. - №5. - С. 224-240.

6. Хубаев Г.Н. Ранжирование объектов по множеству количественных показателей: универсальный алгоритм // РИСК: Ресурсы, информация, снабжение, конкуренция. 2018. - №1. - C. 213-217.

7. Введение в информационный бизнес: Учеб. пособие / О. В. Голосов, С. А. Охрименко, А. В. Хорошилов и др. / Под ред. В. П. Тихомирова, А. В. Хорошилова. - М.: Финансы и статистика, 1996.

8. Хубаев Г.Н. Сокращение бюджетных ассигнований субъектов рынка путем унификации деловых процессов одного назначения// Вестник ЮРГТУ (НПИ). - 2020. №6. - C. 224-232.

9. Хубаев Г.Н. Государство и рынок: как государство может усилить позитивное взаимовлияние субъектов рынка [Электронный pecypc] // Бюллетень науки и практики. 2020. - T. 6. — №6. - C. 175-198. - Peжим доступа: https://doi.org/10.33619/2414$2948 / 55 / 22$.
10. Хубаев Г.Н. Сегментирование рынка на основе различий в требованиях покупателей к функциональной полноте товара: универсальная методика (на примере программных продуктов) // Наука и бизнес: пути развития. - 2019. - №3. - С. 219-224.

11. Хубаев Г.Н. Сравнение сложных программных систем по критерию функциональной полноты // Программные продукты и системы (Software\&Systems). - 1998. №2. - C. 6-9.

12. Хубаев Г.Н. Маркетинг информационных продуктов и услуг: Учебное пособие/ РГЭУ «РИНХ». — Ростов-на-Дону, 2005. — $224 \mathrm{c}$.

13. Хубаев Г.Н. Универсальный метод оптимизации состава характеристик объектов [Электронный ресурс] // Бюллетень науки и практики. - 2019. - Т. 5. - №5. C. 265-275. - Режим доступа: http://doi. org/10.33619/2414-2948/42/35.

14. Хубаев Г.Н. Прогнозирование спроса на товары и услуги: пошаговое уточнение значений с оценкой характеристик распределения // Материалы Международной науч.практ. конф. «Статистика в современном мире: методы, модели, инструменты» (г. Ростов-на-Дону, 26 сентября 2012 г.). — Ростовна-Дону, 2012. - С. 206-211.

15. Хубаев Г.Н. Имитационное моделирование для получения групповой экспертной оценки значений различных показателей// Автоматизация и современные технологии. - 2011. — №11. - С. 19-23.

16. Хубаев Г.Н. Калькуляция себестоимости продукции и услуг: процессно-статистический учет затрат // Управленческий учет. - 2009. — №2. - С. 35-46.

17. Хубаев Г.Н. Сравнение вариантов дизайна объекта: модели и алгоритмы // Вестник Ростовского государственного экономического университета (РИНХ). — 2011. №3. - C. 167-173.

18. Хубаев Г.Н. Проектирование объектов различного назначения: сравнительная оценка вариантов внешнего вида // Содружество (Научный российско-китайский журнал). — 2016. — №8 (7). — С. 76-80.

19. Khubaev G. Assessment of the time required for the acquisition of knowledge // 5 th International Scientific Conference «Applied Sciences and technologies in the United States and Europe: 
common challenges and scientific findings» (New York, USA; February 12, 2014). Section 6. Pedagogy. - New York, 2014. — Pp. 86-90.

20. Хубаев Г.Н. Методика экономической оценки потребительского качества программных средств // Программные продукты и системы (Software\&Systems). - 1995. №1. - C. 2-8.

21. Хубаев Г.Н. Оценка резервов снижения ресурсоемкости товаров и услуг: методы и инструментальные средства // Прикладная информатика. - 2012. - №2 (38). - С. 84-90.

22. Хубаев Г.Н. Информационные и программные системы как объекты активного экспериментирования // Программные продукты и системы (Software\&Systems). 1999. - №2. - C. $2-7$.

23. Хубаев Г.Н. Безопасность распределенных информационных систем: обеспечение и оценка // Известия вузов. Северо-Кавказский регион. Технические науки. Спецвыпуск: Математическое моделирование и компьютерные технологии. - 2002. - С. 11-13.

24. Хубаев Г.Н. Оценка времени вскрытия защиты информационных систем: статистический подход // Проблемы экономики. - 2008. - №6. - С. 135-138.

25. Хубаев Г.Н. Ресурсоемкость продукции и услуг: процессно-статистический подход к оценке // Автоматизация и современные технологии. - 2009. - №4. - С. 22-29.

26. Костин А. М. Модели и методы оценки трудозатрат на вскрытие защиты от копирования рыночных экономических информационных систем: автореф. дис. ... канд. экон. наук: 08.00.13. - Ростов-на-Дону, 2009. - 23 с.

27. Хубаев Г.Н. Расчет совокупной стоимости владения программным продуктом: методическое и инструментальное обеспечение // Вопросы экономических наук. 2010. - №5 (44). - C. 82-87.

28. Хубаев Г., Родина О. Модели, методы и программный инструментарий оценки совокупной стоимости владения объектами длительного пользования (на примере программных систем). Монография. Saarbrucken: LAP LAMBERT Academic Publishing, 2012. - $370 \mathrm{c}$.

29. Хубаев Г.Н., Щербаков С.М., Аручиди Н.А. Программная система анализа сложных систем по критерию функциональной полноты // Свидетельство об официаль- ной регистрации программы для ЭВМ. №2009615296. - М.: Роспатент, 2009.

30. Хубаев Г.Н., Щербаков С.М., Аручиди Н.А., Лубянский В.К. Сравнительная оценка состава операций деловых процессов (СОСОП) // Свидетельство о государственной регистрации программы для ЭВМ. №2017614714. - М.: Роспатент, 2017.

31. Хубаев Г.Н., Щербаков С.М. Система автоматизированного синтеза имитационных моделей на основе языка UML 2.0 (СИМUML 2.0) // Свидетельство о государственной регистрации программы для ЭВМ. №2016661676. - М.: Роспатент, 2016.

32. Хубаев Г.Н., Щербакова К.Н., Сидоренко Д. С. Веб-приложение для сравнительной оценки, анализа динамики и прогнозирования уровня жизни населения субъектов РФ [Электронный ресурс] // Свидетельство о государственной регистрации программы для ЭВМ. - №2019619362. - М.: Роспатент, 2019. - Режим доступа: http://urovenzhizni.ru.

33. Система автоматизированного синтеза имитационных моделей на основе языка UML «CИM-UML» / Авторы-правообладатели: Хубаев Г.Н., Щербаков С.М., Рванцов Ю.А. // СеВІТ 2015 (Ганновер, 2015). Каталог разработок российских компаний. Ministry of Education and Science of the Russian Federation; МСП ИТТ, 2015.

34. Автоматизированный конвертер моделей IDEF0 в диаграммы деятельности языка UML «ToADConverter» («ToADConverter»)/ Авторы-правообладатели: Хубаев Г.Н., Широбокова С.Н., Ткаченко Ю.В., Титаренко Е. В. // CеBIT 2015 (Ганновер, 2015). Каталог разработок российских компаний. Ministry of Education and Science of the Russian Federation; МСП ИТТ, 2015.

35. Информационная система налогового учета БИИНТ-1.0 / Авторы-правообладатели: Хубаев Г.Н., Родина О. В. // СеВIT 2015 (Ганновер, 2015). Каталог разработок российских компаний. - Ministry of Education and Science of the Russian Federation; МСП ИТТ, 2015.

\section{References}

1. Bol'shaja jekonomicheskaja jenciklopedija [The Great Economic Encyclopedia]. - Moscow: Jeksmo, 2007. - 816 p. 
2. Kak uvelichit' spros na rossijskie IT-reshenija na global'nom rynke? [How to increase the demand for Russian IT solutions in the global market?] [Jelektronnyj resurs]. — URL: https:// www.kommersant.ru/doc/3991175.

3. Rynok IT: itogi 2019 [IT market: results of 2019] [Jelektronnyj resurs]. — URL: https:// www.cnews.ru/reviews/rynok_it_itogi_2019.

4. Hubaev G.N. Kak umen'shit' verojatnost' oshibok pri vybore prioritetnyh napravlenij social'nogo i jekonomicheskogo razvitija strany [How to reduce the probability of errors when choosing priority directions of social and economic development of the country] // Bjulleten' nauki i praktiki [Bulletin of Science and Practice]. 2019. - Vol. 5. — №12. - Pp. 265-280.

5. Hubaev G.N. Prognozirovanie dinamiki indikatorov urovnja razvitija jekonomiki strany: modeli, metody, instrumental'nye sredstva (na primere Germanii, Rossii i Shvecii) [Prediction of the dynamics of indicators of the development level of a country's economy: models, methods, tools (for example, Germany, Russia and Sweden)] // Vestnik JuRGTU (NPI) [Bulletin of SRSTU (NPI)]. — 2020. — №5. Pp. 224-240.

6. Hubaev G.N. Ranzhirovanie ob'ektov po mnozhestvu kolichestvennyh pokazatelej: universal'nyj algoritm [Ranking of objects by multiple quantitative indicators: a universal algorithm] // RISK: Resursy, informacija, snabzhenie, konkurencija [RISK: Resources, information, supply, competition]. — 2018. №1. - Pp. 213-217.

7. Vvedenie $\mathrm{v}$ informacionnyj biznes: Ucheb. posobie [Introduction to the information business: Textbook] / O.V. Golosov, S.A. Ohrimenko, A. V. Horoshilov et al. / In V.P. Tihomirov, A. V. Horoshilov (eds.). - Moscow: Finansy i statistika, 1996.

8. Hubaev G. N. Sokrashhenie bjudzhetnyh assignovanij sub'ektov rynka putem unifikacii delovyh processov odnogo naznachenija [Reduction of budget allocations of market entities by unifying business processes for one purpose]// Vestnik JuRGTU (NPI) [Bulletin of SRSTU (NPI)]. - 2020. — №6. - Pp. 224-232.

9. Hubaev G.N. Gosudarstvo i rynok: kak gosudarstvo mozhet usilit' pozitivnoe vzaimovlijanie sub'ektov rynka [The state and the market: how the state can strengthen the positive mutual influence of market subjects] [Jelektron- nyj resurs] // Bjulleten' nauki i praktiki [Bulletin of Science and Practice]. - 2020. — Vol. 6. №6. - Pp. 175-198. - URL: https://doi. org/10.33619/2414-2948/55/22.

10. Hubaev G.N. Segmentirovanie rynka na osnove razlichij $\mathrm{v}$ trebovanijah pokupatelej $\mathrm{k}$ funkcional'noj polnote tovara: universal'naja metodika (na primere programmnyh produktov) [Segmentation of the market on the basis of differences in the requirements of buyers to the functional completeness of the product: a universal methodology (on the example of software products)] // Nauka i biznes: puti razvitija [Science and business: ways of development]. 2019. — №3. - Pp. 219-224.

11. Hubaev G. N. Sravnenie slozhnyh programmnyh sistem po kriteriju funkcional'noj polnoty [Comparison of complex software systems by the criterion of functional completeness]// Programmnye produkty i sistemy [Software products and systems] (Software\&Systems). 1998. — №2. — Pp. 6-9.

12. Hubaev G. N. Marketing informacionnyh produktov i uslug: Uchebnoe posobie [Marketing of information products and services: A textbook] / RGJeU «RINH». - Rostov-on-Don, 2005. - $224 \mathrm{p}$.

13. Hubaev G. N. Universal'nyj metod optimizacii sostava harakteristik ob'ektov [Universal method of optimizing the composition of the characteristics of objects] [Jelektronnyj resurs] // Bjulleten' nauki i praktiki [Bulletin of Science and Practice]. - 2019. - Vol. 5. - №5. - Pp. 265-275. —URL: http://doi.org/10.33619/24142948/42/35.

14. Hubaev G. N. Prognozirovanie sprosa na tovary i uslugi: poshagovoe utochnenie znachenij s ocenkoj harakteristik raspredelenija [Forecasting of demand for goods and services: stepby-step refinement of values with estimation of distribution characteristics] // Materialy Mezhdunarodnoj nauch.-prakt. konf. «Statistika V sovremennom mire: metody, modeli, instrumenty» (g. Rostov-na-Donu, 26 sentjabrja 2012 g.) [Proceedings of the International Scientific and Practical Conference «Statistics in the Modern World: Methods, Models, Tools» (Rostov-onDon, September 26, 2012)]. - Rostov-on-Don, 2012. - Pp. 206-211.

15. Hubaev G.N. Imitacionnoe modelirovanie dlja poluchenija gruppovoj jekspertnoj ocenki znachenij razlichnyh pokazatelej [Simu- 
lation to obtain the group of expert assessment of the values of different indicators] // Avtomatizacija i sovremennye tehnologii [Automation and modern technology]. — 2011. - №11. Pp. 19-23.

16. Hubaev G.N. Kal'kuljacija sebestoimosti produkcii i uslug: processno-statisticheskij uchet zatrat [Calculation of the cost of products and services: process and statistical accounting of costs] // Upravlencheskij uchet [Managerial accounting]. - 2009. — №2. - Pp. 35-46.

17. Hubaev G. N. Sravnenie variantov dizajna ob'ekta: modeli i algoritmy [Comparison of object design options: models and algorithms]// Vestnik Rostovskogo gosudarstvennogo jekonomicheskogo universiteta (RINH) [Bulletin of the Rostov State University of Economics (RSUE)]. — 2011. — №3. - Pp. 167-173.

18. Hubaev G.N. Proektirovanie ob'ektov razlichnogo naznachenija: sravnitel'naja ocenka variantov vneshnego vida [Projecting objects of various purposes: comparative assessment of variants of appearance] // Sodruzhestvo (Nauchnyj rossijsko-kitajskij zhurnal) [Sodruzhestvo (Scientific Russian-Chinese Journal)]. 2016. — №8 (7). - Pp. 76-80.

19. Khubaev G. Assessment of the time required for the acquisition of knowledge // 5th International Scientific Conference «Applied Sciences and technologies in the United States and Europe: common challenges and scientific findings» (New York, USA; February 12, 2014). Section 6. Pedagogy. — New York, 2014. Pp. 86-90.

20. Hubaev G.N. Metodikajekonomicheskoj ocenki potrebitel'skogo kachestva programmnyh sredstv [Methodology of economic assessment of consumer quality of software tools]// Programmnye produkty i sistemy [Software products and systems] (Software\&Systems). 1995. - №1. - Pp. 2-8.

21. Hubaev G. N. Ocenka rezervov snizhenija resursoemkosti tovarov i uslug: metody i instrumental'nye sredstva [Assessment of reserves for reducing the resource intensity of goods and services: methods and tools] // Prikladnaja informatika [Applied Computer Science]. - 2012. - №2 (38). - Pp. 84-90.

22. Hubaev G. N. Informacionnye i programmnye sistemy kak ob'ekty aktivnogo jeksperimentirovanija [Information and software systems as objects of active experimentation]//
Programmnye produkty i sistemy [Software products and systems] (Software\&Systems). 1999. - №2. - Pp. 2-7.

23. Hubaev G.N. Bezopasnost' raspredelennyh informacionnyh sistem: obespechenie i ocenka [Security of distributed information systems: support and evaluation] // Izvestija vuzov. Severo-Kavkazskij region. Tehnicheskie nauki. Specvypusk: Matematicheskoe modelirovanie i komp'juternye tehnologii [News of universities. The North Caucasus region. Technical sciences. Special Issue: Mathematical Modeling and Computer Technologies]. - 2002. - Pp. 11-13.

24. Hubaev G.N. Ocenka vremeni vskrytija zashhity informacionnyh sistem: statisticheskij podhod [Evaluation of the time of opening the protection of information systems: statistical approach] // Problemy jekonomiki [Problems of Economics]. - 2008. — №6. - Pp. 135-138.

25. Hubaev G.N. Resursoemkost' produkcii i uslug: processno-statisticheskij podhod $\mathrm{k}$ ocenke [Resource intensity of products and services: a process-statistical approach to evaluation] // Avtomatizacija i sovremennye tehnologii [Automation and modern technologies]. 2009. — №4. - Pp. 22-29.

26. Kostin A.M. Modeli i metody ocenki trudozatrat na vskrytie zashhity ot kopirovanija rynochnyh jekonomicheskih informacionnyh sistem: avtoref. dis. ... kand. jekon. nauk [Models and methods of estimating labor costs for opening copy protection of market economic information systems: Ph. D. (Economy) Thesis]: 08.00.13. - Rostov-on-Don, 2009. - 23 p.

27. Hubaev G. N. Raschet sovokupnoj stoimosti vladenija programmnym produktom: metodicheskoe i instrumental'noe obespechenie [Calculation of the total cost of ownership of a software product: methodological and instrumental support] // Voprosy jekonomicheskih nauk [Questions of economic sciences]. 2010. — №5 (44). — S. 82-87.

28. Hubaev G., Rodina O. Modeli, metody i programmnyj instrumentarij ocenki sovokupnoj stoimosti vladenija ob'ektami dlitel'nogo pol'zovanija (na primere programmnyh sistem). Monografija [Models, methods and software tools for assessing the total cost of ownership of long-term use objects (on the example of software systems). Monograph]. - Saarbrucken: LAP LAMBERT Academic Publishing, 2012. $-370 \mathrm{~s}$. 
29. Hubaev G. N., Shherbakov S. M., Aruchidi N.A. Programmnaja sistema analiza slozhnyh sistem po kriteriju funkcional'noj polnoty [Software system for the analysis of complex systems according to the criterion of functional completeness] // Svidetel'stvo ob oficial'noj registracii programmy dlja JeVM [Certificate of official registration of the computer program]. №2009615296. - Moscow: Rospatent, 2009.

30. Hubaev G. N., Shherbakov S. M., Aruchidi N.A., Lubjanskij V.K. Sravnitel'naja ocenka sostava operacij delovyh processov (SOSOP) [Comparative evaluation of the operations of the business processes (SOAP)] // Svidetel'stvo o gosudarstvennoj registracii programmy dlja JeVM [Certificate of state registration of a computer program]. — №2017614714. — Moscow: Rospatent, 2017.

31. Hubaev G. N., Shherbakov S.M. Sistema avtomatizirovannogo sinteza imitacionnyh modelej na osnove jazyka UML 2.0 (SIMUML 2.0) [Computer-aided synthesis of simulation models based on UML 2.0 (SIM UML 2.0)] // Svidetel'stvo o gosudarstvennoj registracii programmy dlja JeVM [Certificate of state registration of a computer program]. №2016661676. - Moscow: Rospatent, 2016.

32. Hubaev G. N., Shherbakova K. N., Sidorenko D.S. Veb-prilozhenie dlja sravnitel'noj ocenki, analiza dinamiki i prognozirovanija urovnja zhizni naselenija sub'ektov RF [Web-application for comparative assessment, analysis of dynamics and forecasting of the standard of living of the population of the subjects of the Russian Federation] [Jelektronnyj resurs] // Svidetel'stvo o gosudarstvennoj registracii programmy dlja JeVM [Certificate of state registration of computer programs]. №2019619362. - Moscow: Rospatent, 2019. URL: http://uroven-zhizni.ru.
33. Sistema avtomatizirovannogo sinteza imitacionnyh modelej na osnove jazyka UML «SIM-UML» [System of automated synthesis of simulation models based on the language of the UML language «SIM-UML-models»] / Avtory-pravoobladateli: Hubaev G.N., Shherbakov S.M., Rvancov Ju. A. [Authors-copyright holders: Khubaev G. N., Shcherbakov S.M., Rvantsov Yu. A.] // CeBIT 2015 (Gannover, 2015). Katalog razrabotok rossijskih kompanij [Catalog of developments of Russian companies]. - Ministry of Education and Science of the Russian Federation; MSP ITT, 2015.

34. Avtomatizirovannyj konverter modelej IDEF0 v diagrammy dejatel'nosti jazyka UML «ToADConverter» [Automated converter of IDEF0 models in UML activity diagrams for «ToADConverter»] («ToADConverter») / Avtory-pravoobladateli: Hubaev G. N., Shirobokova S.N., Tkachenko Ju. V., Titarenko E.V. [Authors-copyright holders: Khubaev G.N., Shirobokova S.N., Tkachenko Yu. V., Titarenko E. V.] // CeBIT 2015 (Gannover, 2015). Katalog razrabotok rossijskih kompanij [Cata$\log$ of developments of Russian companies]. Ministry of Education and Science of the Russian Federation [Ministry of Education and Science of the Russian Federation]; MSP ITT, 2015.

35. Informacionnaja sistema nalogovogo ucheta BIINT-1.0 [Information system of tax accounting BIINT-1.0] / Avtory-pravoobladateli: Hubaev G. N., Rodina O.V. [Authors-copyright holders: Khubaev G. N., O Rodina. V.] // CeBIT 2015 (Gannover, 2015). Katalog razrabotok rossijskih kompanij [Catalog of developments of Russian companies]. - Ministry of Education and Science of the Russian Federation [Ministry of Education and Science of the Russian Federation]; MSP ITT, 2015. 


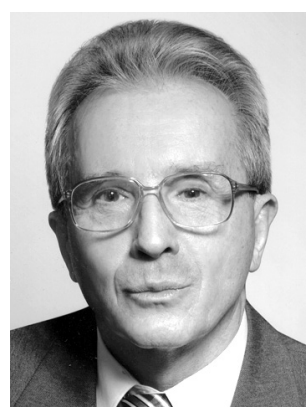

Хубаев Георгий Николаевич - доктор экономических наук, кандидат технических наук, профессор, профессор кафедры Информационных систем и прикладной информатики Ростовского государственного экономического университета (РИНХ).

Khubaev Georgy Nikolaevich - Doctor of Economic Sciences, Candidate of Engineering Sciences, Professor, Professor of the Department of Information Systems and Applied Informatics, Rostov State University of Economics (RSUE).

344002, г. Ростов-на-Дону, ул. Б. Садовая, 69 69 B. Sadovaya st., 344002, Rostov-on-Don, Russia E-mail: gkhubaev@mail.ru 